\title{
RETRACTED ARTICLE: Development of a safe antiparasitic against scuticociliates (Miamiensis avidus) in olive flounders: new approach to reduce the toxicity of mebendazole by material remediation technology using full-overlapped gravitational field energy
}

\author{
Jung-Soo Seo ${ }^{1} \cdot \mathrm{Na}$-Young $\mathrm{Kim}^{2} \cdot$ Eun-Ji Jeon $^{2} \cdot \mathrm{Nam}^{-S i l ~ L e e}{ }^{2} \cdot$ En-Hye Lee $^{3} \cdot$ Myoung-Sug Kim $^{2} \cdot \mathrm{Hak}^{-J e} \mathrm{Kim}^{4}$. $^{2}$ \\ Sung-Hee Jung ${ }^{2}$ (iD
}

Received: 5 March 2018 / Accepted: 6 July 2018 / Published online: 21 July 2018

(C) The Author(s) 2018

This article has been retracted at the request of the Editors-inChief and the Publisher. Post publication peer review has found that the conclusions regarding the phenomenon of full-overlapped gravitational field (FOGF) energy are not supported by evidence.

In addition, the supplementary file has been removed as it was published in error.

All authors agree to this retraction.

Electronic supplementary material The online version of this article (https://doi.org/10.1007/s00436-018-6010-8) contains supplementary material, which is available to authorized users.

Sung-Hee Jung

immu@korea.kr

1 Aquatic Life Disease Control Division, Aquaculture Research Department, National Institute of Fisheries Science, 216,

Gijanghaean-ro, Gijang-eup, Gijang-gun, Busan 46083, Republic of Korea

2 Pathology Research Division, Aquaculture Research Department, NIFS, 216, Gijanghaean-ro, Gijang-eup, Gijang-gun, Busan 46083, Republic of Korea
3 Ministry of Food and Drug Safety, Busan Regional Office of FDS, Center for Food \& Drug Analysis, 65, Sinseon-ro 356 beon-gil, Nam-gu, Busan 48562, Republic of Korea

4 The Asia Pacific Earth-Life Environment Remediation Association, 1494, Yangjae-daero, Gangdong-gu, Seoul 05343, Republic of Korea 